\title{
- SOME COMMENTS ON p-Ps AND DENSITY PARAMETER
}

\author{
T. GOWOREK AND J. WaWryszczuK \\ Instytute of Physics, M. Curie-Skłodowska University \\ Pl. M. Curie-Skłodowskiej 1, 20-031 Lublin, Poland
}

\begin{abstract}
The constraint-free analysis of several representative positron lifetime spectra was performed with the resolution time of $144 \mathrm{ps}$. The role of p-Ps parameters in understanding of the positronium structure in solids is discussed.
\end{abstract}

PACS numbers: $36.10 . \mathrm{Dr}$

When the positrons annihilate in a medium in which positronium atoms can be formed, the $e^{+}$lifetime spectrum contains at least three distinct components: the one related to annihilation in collisions, the second "long-lived" produced by decaying ortho-Ps atoms (that one can be of a complex structure, splitting into two components, or rather continuous distributions); the third component, the shortest-lived one, belongs to singlet para-Ps. Due to its low intensity and short lifetime the p-Ps component is hardly separable from the remainder of the spectrum: it is superposed on the top of broader distribution smeared out by the finite time resolution of measuring system. Thus, very often, to facilitate the processing of complex lifetime spectra one introduces some constrains concerning this component: fixing the lifetime at 124 ps as expected for p-Ps in vacuum, or fixing the ratio of intensities ortho-to-para equal 3 , according to statistical weights of respective states.

Fixing the p-Ps lifetime at $124 \mathrm{ps}$ is not justified. For p-Ps annihilating in the medium the decay rate constant is

$$
\lambda_{\mathrm{s}}=\lambda_{\mathrm{s}_{\mathrm{i}}}+\lambda_{\mathrm{po}}
$$

where $\lambda_{\mathrm{s}_{\mathrm{i}}}$ - intrinsic decay rate of singlet state, $\lambda_{\mathrm{po}}$ - pick-off rate. Both values appearing in Eq. (1) are difficult to determine.

(1) The question arises: Is the pick-off rate for singlet and triplet positronium identical? Theoretical considerations by Dupasquier et al. [1] lead to the conclusion that $\lambda_{\text {po }}$ should be independent on Ps spin state. If so, the $\lambda_{\text {po }}$ term increases the decay rate by about $1 \mathrm{~ns}^{-1}$ (for typical solid).

(2) The value of $\lambda_{s_{i}}$ is not identical with that in vacuum. The experiments with positronium quenching by magnetic field show that local electron density in positron site is usually smaller than in vacuum ("swollen Ps"), thus

$$
\lambda_{\mathrm{si}_{\mathrm{i}}}=\eta^{\prime} \lambda_{\mathrm{s}}(\mathrm{vac}) \text {. }
$$


$\lambda_{\mathrm{s}}(\mathrm{vac})=8 \mathrm{~ns}^{-1} ; \eta^{\prime}$ is called density parameter. Both $\lambda_{\text {po }}$ and $\eta^{\prime}$ modify the p-Ps lifetime.

There is to note that "density parameters" $\eta, \eta^{\prime}$ describing the spatial extension of Ps wave function play an important role in the estimations of void sizes from o-Ps lifetime. The well-known formula describing $\tau_{3}$ as a function of void radius is essentially an expression for the overlap of $P s$ wave function with electronic layer around the void. A modified wave function must be taken into account in any serious void distribution modelling.

The rule for intensities ortho/para equal 3 should be generally fulfilled. Nevertheless, one can expect that situation can be more complex. If the value of $\eta^{\prime}$ density parameter is very low, the p-Ps lifetime can be even longer than $\tau_{2}$ (i.e. free annihilation) and fall among the long-lived components. E.g. in the case of liquid dimethylformamide [2] at least for a part of Ps atoms $\eta^{\prime}=0.13$ was observed; that corresponds to the lifetime $\tau_{1}=680$ ps! Thus, this part of p-Ps intensity disappears from the classic "shortest-lived component".

It seems that precise and reliable data about p-Ps parameters can be very valuable for our knowledge of Ps structure in condensed media. A constraint-free analysis of the spectrum, in which the p-Ps component can be precisely separated from the remainder would be very advisable. One of the conditions to prepare such a spectrum is a maximal reduction of resolving time of the spectrometer.

To gain the information (without excessive expectation) how close we can approach to reliable determination of p-Ps lifetime and intensity, we modified our spectrometer. Using the dynode output from XP2020Q tubes coupled to $\varnothing 1^{\prime \prime}$, $h=0.5^{\prime \prime}$ NE111 plastics we got the FWHM of prompt curve equal $144 \mathrm{ps.} \mathrm{The}$ shape of the curve was approximated by two gaussians (using RESOLUTION program), the spectra containing $\approx 3 \times 10^{6}$ pulses were analysed by POSITRONFIT [3]. The source of $5 \mu \mathrm{Ci}^{22} \mathrm{Na}$ was deposited in a kapton envelope. Typical samples selected to analyse their $\mathrm{e}^{+}$lifetime spectra were: a polymer - high density polyethylene (HDPE), simple solid with long-lived component existing at all temperatures - solid $n$-octacosane, and mixed crystal with positronium formed at the well-defined defects - p-terphenyl $+0.4 \%$ anthracene.

The spectrum of HDPE was approximated by 4 exponentials, the results are given in Table I. In the case of $n$-octacosane in its room temperature phase the four-component fit was not easy to perform. The values for long-lived components

TABLE I

High density polyethylene spectrum.

\begin{tabular}{l|c|c|c|c|c}
\hline \hline Lifetimes, ps & $124 \pm 6$ & $355 \pm 8$ & $990 \pm 125$ & $2523 \pm 47$ & Variance \\
\cline { 1 - 5 } Intensities, \% & $12.7 \pm 1.1$ & $61.1 \pm 0.8$ & $8.7 \pm 0.8$ & $17.6 \pm 0.8$ & 0.932
\end{tabular}

are loaded with large error (overlapping error bars for $\tau_{3}$ and $\tau_{4}$ !), depend on details of prompt curve, thus also the three-component fit was done (see Table II). This can mean that we have a distribution of long lifetimes (with one maximum), or the spectrum is distorted by weak very long-lived component belonging to Ps 
TABLE II

Octacosane spectrum.

\begin{tabular}{l|c|c|c|c|c}
\hline \hline Lifetimes, ps & $119 \pm 11$ & $331 \pm 9$ & $1000 \pm 339$ & $1727 \pm 509$ & Variance \\
\cline { 1 - 4 } Intensities, \% & $8.0 \pm 1.4$ & $69.5 \pm 1.1$ & $13.0 \pm 8.8$ & $9.4 \pm 10.6$ & 1.064 \\
\hline Lifetimes, ps & $150 \pm 7$ & $356 \pm 4$ & $1402 \pm 8$ & & Variance \\
\cline { 1 - 4 } Intensities, \% & $13.3 \pm 1.1$ & $67.7 \pm 1.0$ & $19.0 \pm 0.2$ & & 1.137
\end{tabular}

in the vicinity of structural conformers [4], neglected in our analysis. In a mixed crystal it was impossible to fit 4 components with $I_{i}, \tau_{i}$ values having physically reasonable meaning, thus the number of components was reduced to 3 (Table III).

\section{TABLE III}

Spectrum of $\mathrm{p}$-terphenyl $+0.4 \%$ antracene.

\begin{tabular}{l|c|c|c|c}
\hline \hline Lifetimes, ps & $143 \pm 8$ & $311 \pm 4$ & $1461 \pm 10$ & Variance \\
Intensities, \% & $13.9 \pm 1.7$ & $69.1 \pm 1.6$ & $17.0 \pm 0.2$ & 1.065
\end{tabular}

The lifetime of p-Ps in HDPE was found quite close to its vacuum value. Existing data on the magnetic quenching [5] indicate that $\eta^{\prime}$ for the fourth component in polyethylene is less than 1 , while for the third one, on contrary, $\eta^{\prime}>1$, thus this experimental result for $\tau_{1}$ can be consistent with magnetic data. The $\eta\left(=\eta^{\prime}\right)$ value for our mixed crystal is known [6] as $0.80 \pm 0.02$, thus expected $\tau_{1}=(141 \pm 4) \mathrm{ps}$ coincides with the measured one. If the $\eta^{\prime}$ value for $n$-octacosane is the same as for shorter-chain $n$-octadecane [7] the expected $\tau_{1}$ is about $133 \mathrm{ps}$, thus somewhere between the fitted values in the three- and four-component analysis.

It looks optimistic, but it is easy to see that the intensity ratio $I_{3}\left(+I_{4}\right): I_{1}$ is systematically less than 3 , closing rather to 2 . Fixing this ratio at 3 one receives poorer variance and $\tau_{1}$ falls to about $100 \mathrm{ps}$. The attempt to modify the proportions of gaussians forming the prompt curve between $10 \%$ and $30 \%$ (this is not the fitting parameter) brings no improvement of $I_{1}$ intensity value (changes by $\approx 1 \%$ ). With the single gaussian prompt curve the discussed ratio is further greatly reduced.

In the case of polymer and, may be, paraffin (octacosane) one can explain the observed excessive $I_{1}$ by an oversimplified model of the spectrum: the long-lived part, being a continuous (gaussian-like?) distribution of lifetimes is here approximated by a single "mean" exponential. This approximation leaves in the range of short delays an excess of counts artificially lifting the shorter lived components. However, in our mixed crystal, where all the data suggest the existence of single kind of Ps trapping sites and pure single exponential (see failure of 4-component fit), the arguments used above are not applicable. And in just that case the ortho/para ratio deviates mostly from statistical weights. Unfortunately, we have no explanation of this result. Of course, we are aware of limited reliability of listed data, the errors shown are the fitting ones only. Nevertheless it seems worthwhile 
to continue the efforts to reduce the resolution (e.g. new fast scintillators of small size) and do not neglect the potential information contained in p-Ps properties.

\section{Conclusion}

The density parameter $\eta$, introduced in the magnetic quenching experiments, and known for several randomly selected media only, deserves more attention due to its universal character: it modifies p-Ps-lifetime, the triple coincidence decay intensity, influences the estimation of positronium trap sizes, etc.

The highest available resolutions of lifetime spectrometers seem to be close to the value enabling reliable p-Ps lifetime and intensity measurements.

\section{References}

[1] A. Dupasquier, P. De Natale, A. Rolando, Phys. Rev. B 43, 10036 (1991).

[2] I. Billard, J. Ch. Abbé, G. Duplâtre, Chem. Phys. 184, 365 (1994).

[3] P. Kirkegaard, N.J. Pedersen, M. Eldrup, Risø Report M2740, Risø National Laboratory, Denmark 1989.

[4] T. Goworek, W. Górniak, Mater. Sci. Forum 105-110, 1565 (1992).

[5] G. Consolati, F. Quasso, Appl. Phys. A 50, 43 (1990).

[6] T. Goworek, A. Badia, G. Duplâtre, J. Chem. Soc. Faraday Trans. 90, 1501 (1994).

[7] G. Consolati, N. Gambara, F. Quasso, Acta Universitatis Wratislaviensis, Ser. Mat., Fiz., Astronom. 59, 33 (1991). 